\title{
Primary intracranial leiomyosarcoma presenting with frontal bone mass: a case report
}

\author{
Shaghayegh Kamian', Abdolali Ebrahimi ${ }^{2}$ Kaveh Ebrahim Zadeh ${ }^{3}$, Behnaz Behzadi ${ }^{4}$ \\ 'Department of Radiotherapy Oncology, Imam Hossein Hospital, Shahid Beheshti University of Medical Sciences, Tehran, Iran \\ ${ }^{2}$ Department of Pathology, Imam Hossein Hospital, Shahid Beheshti University of Medical Sciences, Tehran, Iran \\ ${ }^{3}$ Department of Neurosurgery, Skull Base Research Center, Loghman Hakim Hospital, Shahid Beheshti University of Medical Sciences, Tehran, \\ Iran \\ ${ }^{4}$ Department of Radiation Oncology, Imam Hossein Hospital, Shahid Beheshti University of Medical Sciences, Tehran, Iran
}

Received: August 11, 2020

Revised: October 24, 2020

Accepted: November 9, 2020

Correspondence:

Behnaz Behzadi

Department of Radiation Oncology, Imam Hossein Hospital, Shahid

Beheshti University of Medical

Sciences, Tehran, Iran

Tel: +989122590051

E-mail: behnaz_bzd@yahoo.com

ORCID:

https://orcid.org/0000-0003-4135-7148
Primary intracranial mesenchymal neoplasms are rare tumors. These tumors are usually metastatic disease from other primary sites. We presented a 31-year-old man with a 6-month history of gradually enlarging frontal mass and positional headache. There was no other symptom demonstrating other organs' involvement. The patient underwent an uncomplicated craniotomy with clear surgical margins. The pathology review and the immunohistochemistry staining confirmed leiomyosarcoma grade II. We prescribed radiation therapy with tumor dose of $60 \mathrm{~Gy}$ in 30 fractions with conformal treatment planning to the tumor bed. As this disease has a high potency for metastasis, we advised four courses of single agent doxorubicin chemotherapy $75 \mathrm{mg} / \mathrm{m}^{2}$ every 4 weeks starting one month after the end of radiotherapy. In the last follow-up visit 34 months later, the patient was disease free in physical exam and imaging findings.

Keywords: Brain neoplasms, Intracranial, Leiomyosarcoma

\section{Introduction}

Primary intracranial mesenchymal neoplasms are rare tumors. The majority of intracranial leiomyosarcomas are usually metastatic disease from other primary sites. The common sites of origin are gastrointestinal tract or reproductive system especially in women [1].

Leiomyosarcoma (LMS) as a primary intracranial tumor arise from blood vessels or dura matter mesenchymal cells. These tumors can mimic meningioma on imaging. Primary intracranial LMS is much rare tumor and we could find 16 reported cases till 2018 [1] and two cases in 2019 [2,3] and three cases in 2020 [4-6]. It has been reported to be less than $1 \%$ of brain biopsy that was about 3 out of 25,000 primary brain tumors [2].

There are some predisposing factors related to this pathology including radiation exposure [7], immunocompromised states [8], and infections with human immunodeficiency virus (HIV) [9] or Epstein-Barr virus (EBV) [10]. Although there are some reports that this tumor has been seen in immunocompromised patients especially in AIDS [11], the incidence in immune-deficient patients is rare, in general [12]. There are some reports that this pathology has been found in cases who had a history of lung or renal transplantation $[8,12]$. Also, immunosuppression and radiation induced tumorigenesis have been proposed as probable causative factors for the development of primary intracranial LMS [13].

The symptoms are usually headache, gait disturbance, hemiparesis or seizures. Sometimes this tumor presents with sudden intratumoral hemorrhage [1]. Immunohistochemical staining (IHC) is a helpful tool for discriminate this pathology from the metastasis of other organs or meningioma.

The prognosis of this tumor is poor and the survival range from 3 
weeks [14] to 44 months [15] in the literature. A standard protocol for the treatment of this disease could not be determined due to the limited number of cases. Surgery remains the mainstay of treatment with the attempt to remove total resection. Radiotherapy and chemotherapy have been used in multiple cases. There are some reports about the use of Gamma Knife radiosurgery in combination with partial surgery with a survival of 3 months [13]. There is limited experience with chemotherapy in this disease [16].

We present a very rare case of primary intracranial LMS who had not immunosuppression and has survived 34 months without any recurrence until the report of this paper despite the poor prognosis reported in the literature.

\section{Case Report}

Written informed consent was obtained and signed on the admission of the patient and was kept in his medical files. He declared that his medical information can be used for research purposes in the future and also agree with the provided treatment options.

The patient was a 31-year-old male with a gradually enlarging mass in frontal bone and positional headache, since 6 months before admission. He was visited for the first time in November 2017. There was no other symptom demonstrating other organs' involvement. He did not have any positive note in his past medical and habitual history. In the physical examination, there was a palpable fixed and non-tender mass measuring approximately $3 \mathrm{~cm} \times 2 \mathrm{~cm}$ over the left frontal region with no ulcer on the skin. Also, he had small multiple firm, non-tender subdermal lesions on the extremities and trunk compatible with lipoma.

We check blood tests which included complete blood counts and biochemistry which were normal. Also, serology tests for HIV, hepatitis-B virus (HBV), hepatitis-C virus ( $\mathrm{HCV})$, and EBV were all negative. In the computerized tomography (CT) scan of the brain, there was a destructive enhancing soft tissue mass measuring $35 \mathrm{~mm} \times$ $22 \mathrm{~mm}$ in left frontal bone and enhancement of the adjacent meninges. The brain magnetic resonance imaging (MRI) showed a 34 $\mathrm{mm} \times 28 \mathrm{~mm}$ left frontal skull vault tumoral lesion with high signal intensity in T2 which showed avid enhancement of adjacent dura (Fig. 1). The differential diagnosis with these features in pathology were metastasis, solitary plasmacytoma, hemangiopericytoma and angiosarcoma.

An uncomplicated surgery including mass resection through craniotomy and subsequent cranioplasty was performed on November 13,2017 . The histologic examination demonstrated a high-grade spindle cell neoplasm with invasion of bone and fibro adipose tissue. In the pathologic report, dura matter involvement without adhesion to the bony skull was noted. The surgical margins were tu- mor free. In the second pathology opinion the diagnosis was high grade pleomorphic sarcoma compatible with anaplastic meningioma. The tumor composed of interlacing bundles of spindle to oval cell with moderate pleomorphism and noticeable mitotic figures (Fig. 2).

In IHC, the tumor was desmin, $\mathrm{h}$-caldesmon, and vimentin positive but immunostaining for epithelial membrane antigen (EMA), progesterone receptor (PR), glial fibrillary acidic protein (GFAP), thyroid transcription factor-1 (TTF-1), calcitonin, melan $A$, human melanoma black 45 (HMB45), smooth muscle actin (SMA), CD34 and S100 were negative (Figs. 3, 4). The last pathology review and the IHC confirmed leiomyosarcoma grade II.

Following surgery, the patient was referred for radiotherapy to our radiation oncology center. The patient underwent staging workup including chest, abdomen and pelvic CT scan and also

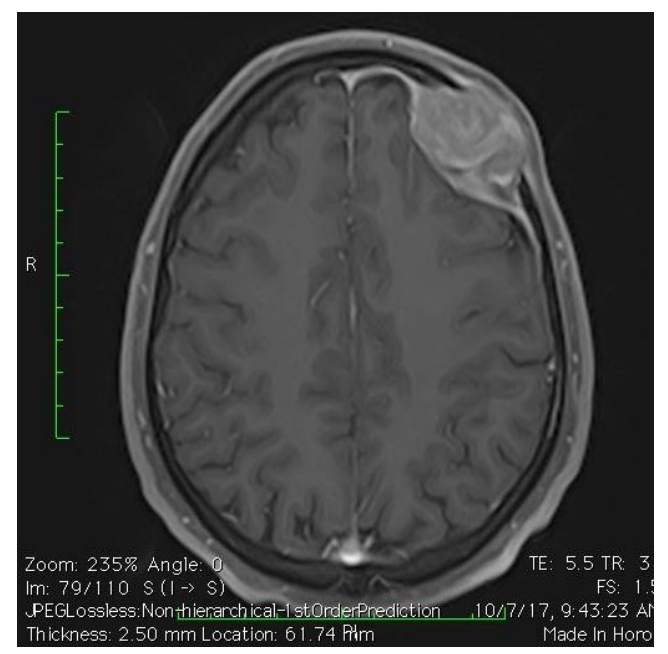

Fig. 1. An axial view of brain magnetic resonance imaging.

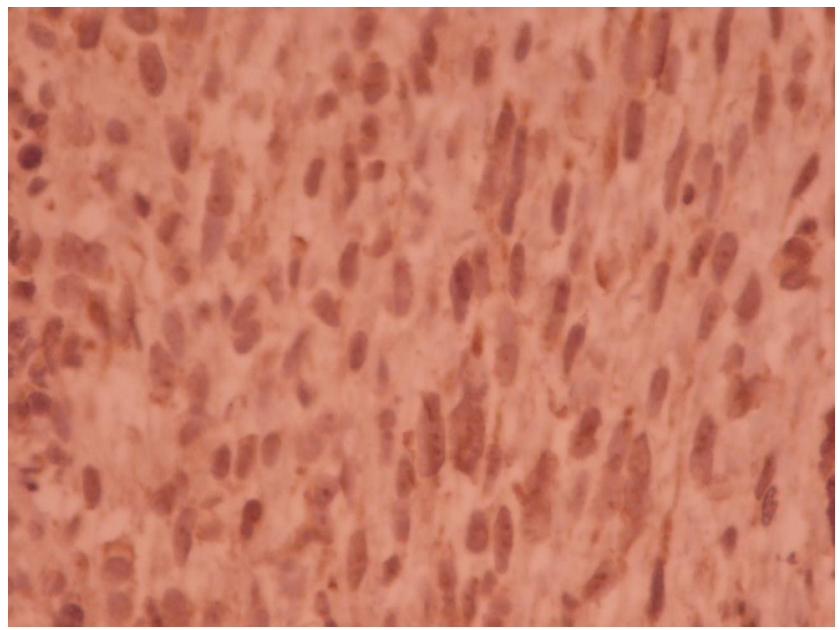

Fig. 2. Immunohistochemistry staining showing h-caldesmon positivity (microscopic image at $\times 40$ ). 


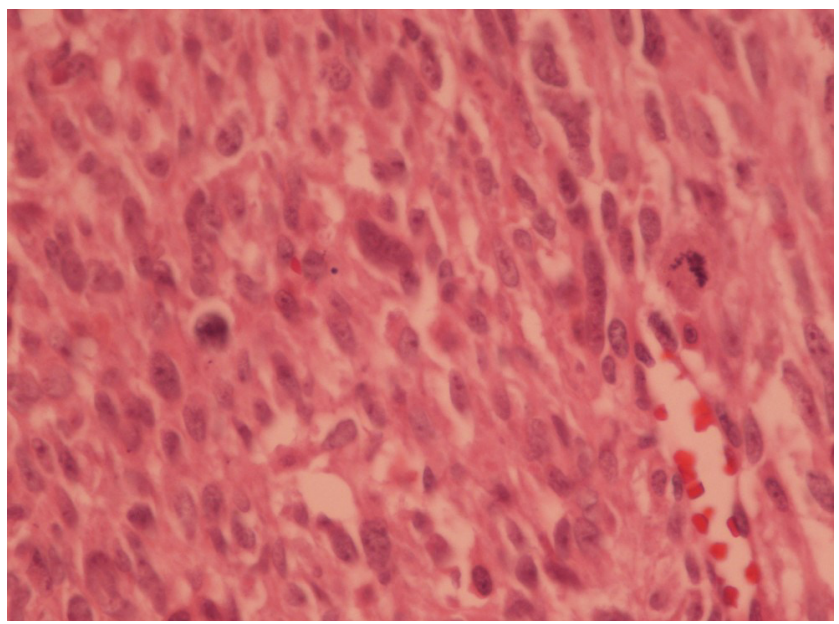

Fig. 3. Interlacing bundles of spindle to oval cells with moderate dysplasia and noticeable mitotic figures (microscopic image at $\times 40$ ).

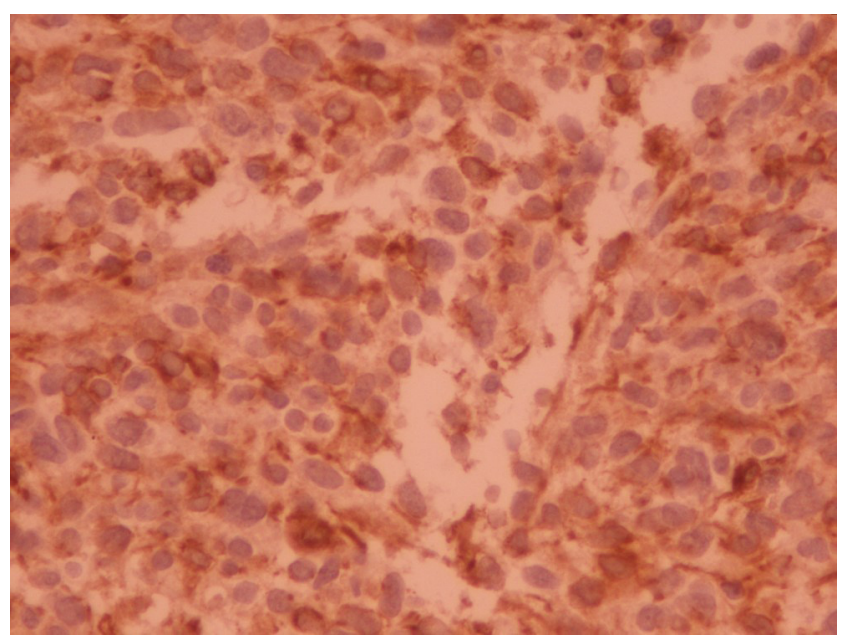

Fig. 4. Immunohistochemistry staining showing vimentin positivity (microscopic image at $\times 40$ ). whole-body bone scan. None of the organs outside brain and skull were involved. Also, there was no residue in brain MRI after surgery.

The brain MRI before and after the surgery were fused in the CT simulation and targets were define respect to mass and tumor bed. The isodoses for target volumes are illustrated in Fig. 5. Gross tumor volume (GTV) is in red and planning target volume (PTV) is in blue. We added $1.5-\mathrm{cm}$ margins around the GTV to make clinical target volume (CTV) and $0.5 \mathrm{~cm}$ more to define PTV. Treatment planning system was ISOgray edition 4.2.3 (DOSIsoft, Paris, France), and $63 \mathrm{~L}$ for conformal treatment planning. Total radiation dose was $60 \mathrm{~Gy}$ in 30 fractions.

As this disease has a high potency for metastasis, we advised four cycles of single agent doxorubicin chemotherapy $75 \mathrm{mg} / \mathrm{m}^{2}$ every 4 weeks starting one month after the end of radiotherapy.

The patient was followed as a common brain tumor with brain MRI every 3 months. But chest, abdomen and pelvic CT scan were also requested every 6 months. In the last visit in August 2020, the patient did not have symptom and sign of disease in the physical examination and also, there was no evidence of disease local recurrence or metastasis in imaging.

\section{Discussion}

The prevalence of bone and soft tissue sarcomas is four in 100,000 people per year [17]. LMS is found more frequent in uterus, stomach, intestines or walls of blood vessels. Leiomyosarcomas represent 1\%-4\% of soft tissue sarcomas of the head and neck [5]. Involvement of central nervous system (CNS) is very infrequent and most of the cases diagnosed as intracranial LMS correspond to

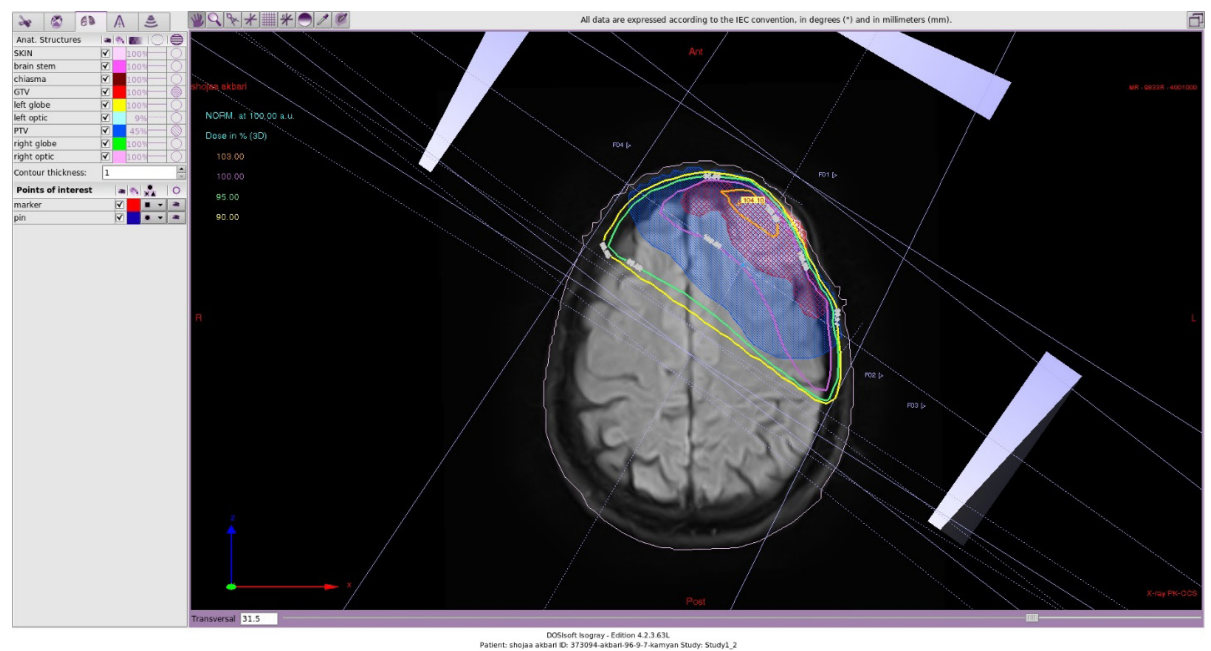

Fig. 5. Target volumes (GTV in red and PTV in blue) and isodoses around target volume (isodose 95\% in green and 90\% in yellow with maximum hot dose of 105\% in the GTV). GTV, gross tumor volume; PTV, planning target volume. 
metastatic disease of other primary sites such as the gastrointestinal tract or uterus $[5,18,19]$.

Primary LMS of CNS is extremely rare with reported approximately 21 cases in the world till mid-2020. These tumors can originate from mesenchymal cells in dura matter or blood vessels of brain [16]. There has been an increase in its incidence and reports in recent years, probably associated with HIV infection and better pathological diagnosis of the cases [5]. Sarcoma develops in some patients with brain tumor after radiotherapy or chemotherapy [19]. Previous studies have also reported that LMS is associated with immunodeficiency states caused by EBV or HIV infections $[18,20]$. The case we reported had no sign or symptom of immunosuppression and the laboratory findings were also all negative for EBV, HIV.

Based on review of Gautam and Meena [12], surgery with negative surgical margins remained the primary treatment of intracranial LMS. Complete resection of the tumor was possible in majority of the reported cases, and it was associated with improved survival rate. Also, improving local control of the tumor has been reported by using radiation therapy after surgery [12]. In recent studies, Gamma Knife radiosurgery and stereotactic robotic cyber knife radiosurgery have been used for intracranial LMS $[12,13]$.

There are also some reports about the use of chemotherapeutic agents for this disease because of high propensity for distant metastasis; however, results were inconsistent [12].

Based on a case report, a 19-year-old man who had no metastasis and normal serology finding underwent craniotomy with negative margins and then he received radiotherapy. No recurrences occurred after 18 months of follow-up [19].

Also, there was another case who was a 26-year-old man with primary intracranial LMS and no evidenced of metastasis. He had complete surgical resection and received post-operative radiotherapy with total dose of $61.8 \mathrm{~Gy}$ in 34 fractions. The patient declined chemotherapy. After 5 months of follow-up, liver and lung metastasis developed. The patient received liposomal doxorubicin with no response and died after 2 months [16].

Although most of the reported cases had surgery and post-operation radiotherapy, there is a report which the tumor was only removed by surgery. The was no recurrences despite the patient received no other treatment till the report of the paper [1].

There is a report of a man with a 4-year history of a temporo-occipital bump and disturbance with reading words. He had a sudden onset of headache in occipital region and evaluation revealed a temporal osteolytic mass lesion extending into the subcutaneous layer. After surgical removal of mass, local radiation therapy of 60 Gy was prescribed. But due to a left subdural hematoma and intrasylvian subarachnoid hemorrhage 3 months later and another temporal subcortical hemorrhage 2 months later, the patient died [3].

There is a report about late brain tumor recurrence by Maslehaty et al. [17] in 2016. They reported a 43-year-old man with the history of resected astrocytoma grade 1 in right cerebellar hemisphere 10 years before. He had another mass in right occipital lobe which was completely resected, and the pathology was LMS. They found no evidence of extracranial involvement. He received post-operative radiotherapy (50 Gy) and HDR brachytherapy (20 Gy). They reported 6 months of follow-up and there was no recurrence in the bed of tumor or distant metastasis [17].

A recent case report introduced a 23-year-old man with a history of EBV and HIV infection who had a lesion in the cavernous sinus. Only excisional biopsy was performed which was low grade LMS. Due to the mass location, complete resection could not be performed and the patient was treated with radiotherapy. In a short follow-up of 6 months after treatment, the patient had stable disease and was asymptomatic [5].

In our presented case, operation was performed with no residue and radiotherapy and systemic chemotherapy were prescribed. Although the reported cases in the literature had survival from 3 weeks to 44 months, the presented case was alive till the report and disease free for 34 months from the diagnosis and surgery. It might be due to the location of tumor which could be removed safely and also adjuvant radiotherapy and chemotherapy which decrease the incidence of local recurrence and systemic metastasis.

In conclusion, primary intracranial LMS is a very rare tumor. There is an association between these neoplasm and HIV and EBV in most of the cases. The diagnosis is based on histopathologic findings and IHC. Surgical resection is the main part of treatment. Prognosis of this neoplasm is poor due to the high propensity for local and distant recurrences. It seems that complete resection of tumor can help in local control of disease. In the review of literature and case reports, there were other treatment options too; such as radiotherapy and chemotherapy. Although the role of adjuvant chemotherapy is still on debate, some reports used it in the setting of distant metastasis.

\section{Conflicts of interest}

No potential conflict of interest relevant to this article was reported.

\section{References}

1. Torihashi K, Chin M, Yoshida K, Narumi O, Yamagata S. Primary intracranial leiomyosarcoma with intratumoral hemorrhage: case report and review of literature. World Neurosurg 2018;116:169- 
73.

2. Li $X L$, Ren J, Niu RN, et al. Primary intracranial leiomyosarcoma in an immunocompetent patient: case report with emphasis on imaging features. Medicine (Baltimore) 2019;98:e15269.

3. Saito A, Ninomiya A, Ishida T, et al. Intractable repeated intracerebral hemorrhage due to primary dural leiomyosarcoma: case report and literature review. World Neurosurg 2019;122:116-22.

4. Bregy A, Lim J, Lohman R, et al. Primary leiomyosarcoma of the calvarium with intracranial extension: a case report. Indian J Surg Oncol 2020 Jul 1 [Epub]. https://doi.org/10.1007/s13193020-01129-z.

5. Morales RL, Alvarez A, Norena MP, Torres F, Esguerra J. Lowgrade leiomyosarcoma of the cavernous sinus in an HIV positive patient: case report. Cureus 2020;12:e6758.

6. Sadeh M, Chaudhry NS, Selner A, Behbahani M, Valyi-Nagy T, Atwal G. Intracranial leiomyoma associated with Epstein-Barr virus: a cerebellopontine angle mass presenting with trigeminal neuralgia. World Neurosurg 2020;141:284-90.

7. Niwa J, Hashi K, Minase T. Radiation induced intracranial leiomyosarcoma: its histopathological features. Acta Neurochir (Wien) 1996;138:1470-1.

8. Patel U, Patel N. Primary intracranial leiomyoma in renal transplant recipient. Saudi J Kidney Dis Transpl 2017;28:921-4.

9. Ritter AM, Amaker BH, Graham RS, Broaddus WC, Ward JD. Central nervous system leiomyosarcoma in patients with acquired immunodeficiency syndrome: report of two cases. J Neurosurg 2000;92:688-92.

10. Takei H, Powell S, Rivera A. Concurrent occurrence of primary intracranial Epstein-Barr virus-associated leiomyosarcoma and Hodgkin lymphoma in a young adult. J Neurosurg 2013;119:499-
503.

11. Sivendran S, Vidal Cl, Barginear MF. Primary intracranial leiomyosarcoma in an HIV-infected patient. Int J Clin Oncol 2011;16:636.

12. Gautam S, Meena RK. Primary intracranial leiomyosarcoma presenting with massive peritumoral edema and mass effect: Case report and literature review. Surg Neurol Int 2017;8:278.

13. Zhang $H$, Dong $L$, Huang $Y$, et al. Primary intracranial leiomyosarcoma: review of the literature and presentation of a case. Onkologie 2012;35:609-16.

14. Aeddula NR, Pathireddy S, Samaha T, Ukena T, Hosseinnezhad A. Primary intracranial leiomyosarcoma in an immunocompetent adult. J Clin Oncol 2011;29:e407-10.

15. Saito N, Aoki K, Hirai N, et al. Primary intracranial leiomyosarcoma of the cavernous sinus: case report. Austin J Med Oncol 2014;1:2.

16. Hussain S, Nanda A, Fowler M, Ampil FL, Burton GV. Primary intracranial leiomyosarcoma: report of a case and review of the literature. Sarcoma 2006;2006:52140.

17. Maslehaty $H$, Nabavi $A$, Mehdorn HM. Primary intracranial leiomyosarcoma: case report and principles for treatment. J Neurol Neuromed 2016;1:16-20.

18. Bejjani GK, Stopak B, Schwartz A, Santi R. Primary dural leiomyosarcoma in a patient infected with human immunodeficiency virus: case report. Neurosurgery 1999;44:199-202.

19. Alijani B, Yousefzade S, Aramnia A, Mesbah A. Primary intracranial leiomyosarcoma. Arch Iran Med 2013;16:606-7.

20. Brown HG, Burger PC, Olivi A, Sills AK, Barditch-Crovo PA, Lee RR. Intracranial leiomyosarcoma in a patient with AIDS. Neuroradiology 1999;41:35-9. 